\title{
Automated System for Measurement and Characterization of Planar Active Arrays
}

\author{
M. A. Salas Natera, R. Martínez Rodríguez-Osorio, L. de Haro Ariet and J. L. Fernández-Jambrina, \\ Universidad Politécnica de Madrid, Madrid, Spain, (msalasn; ramon; leandro; jambrina@gr.ssr.upm.es)
}

\begin{abstract}
This work has been focused to describe the development and implementation of an automated system for measurement and characterization of planar active arrays. This automated system is capable of reducing time and operative cost and it is based on multilayer hardware architecture and the control software. The motivation of this work is the calibration of one triangular active array panel of the Geodesic Antenna Array GEODA. The measurements and characterization are complicated and multipart tasks in the design, development and calibration of active antenna arrays. In this paper an automated system is presented supported by measurements and characterization of one planar active array.
\end{abstract}

\section{INTRODUCTION}

Nowadays, earth stations have as a common feature the use of large reflector antenna for downloading data from satellites [1]. However, large reflectors have a number of impairments as their mechanical complexity, low flexibility, and high cost. Furthermore, the download capacity of existing earth station will be saturated due to the launch of several new satellites [2] and [3]. As a consequence, this capacity must be increased to achieve the satellite communication requirements and the feasibility of other antenna technologies must be evaluated, such as conformal adaptive antennas based on multiple planar active arrays.

In the scenery under study, the capability to track several satellites simultaneously, higher flexibility, lower production and maintenance cost, modularity and a more efficient use of the spectrum; are the most important advantage to boost up antenna arrays over large dishes. In this work the antenna under test (AUT) is a planar active array as a part of one geodesic adaptive antenna, which has been designed as a first version to receive signals at 1.7 GHz. Subsequently, in recent efforts the system has been upgraded also for transmission [4]. This antenna uses adaptive beamforming algorithms based on spatial reference to track LEO satellites, and the correct performance of the beamformer depends on the measurement and characterization in order to apply an appropriate calibration procedure.

Since the AUT is a planar active array, in the calibration process there are a number of errors due to manufacturing and gradual changes as a result of the thermal variation and the aging of components, which must be consider. As an example of an error categorization there are: the Sensor
Mutual Coupling, Sensor Location, Sensor Gain, Sensor Phase Errors as static errors; and the Sensor Amplifier, Sensor Phase Shifter and Sensor Temperature Error, as dynamic errors. In order to compensate the degradation due to these errors, intensive calibration techniques must be implemented after the measurement and characterization of the active antenna array. Finally, the AUT measurement requirements are those owing to the calibration procedure.

It is well known that the calibration process is quite expensive in time and operative cost [5] and [6]. Thus, the development and implementation of an automated system to achieve the requirement in the laboratory and in the anechoic chamber is a much valuable solution for costs reduction.

The calibration process presented in Figure 1 of the AUT consist of an Off-line calibration procedure to compensate statics errors, an On-line calibration procedure to compensate dynamic errors; and an On-site calibration procedure added to compensate the change of the work frequency for transmission. This exhaustive calibration process requires a number of measurements classified as follows: First, an S coupling parameter measurement must be done to model the mutual coupling effect in order to define a coupling compensation model. Second, an $\mathrm{S}_{21}$ parameter measurement of the RF circuits must be done for the characterization of these circuits. Third, the Test signal measurement for calibration of the RF circuits in On and Off-line operating settings. Finally, the anechoic chamber measurements of the active antenna array for the Off-line calibration and characterization.

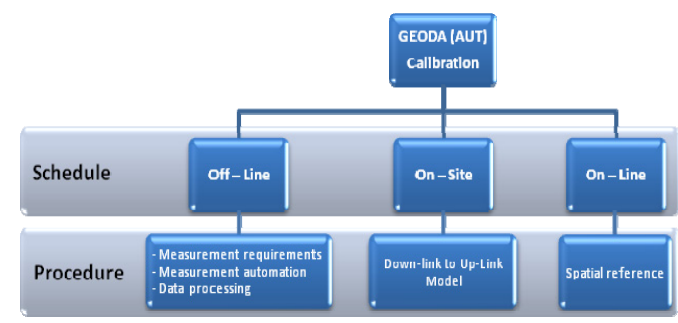

Figure 1 - Calibration process of the AUT

In this work, we present an automated system for the last three groups of measurements described above capable of reducing time and operative cost. This automated system for measurements is based on multilayer hardware architecture and the control software. 
The paper is organized as follows. Section II introduces the antenna under test and its control system. Section III describes the automated measurements. Section IV presents a discussion about the more relevant results. Finally, section $\mathrm{V}$ draws the conclusions of this paper.

\section{Antenna Under Test}

\section{Active antenna array}

For this contribution the AUT is one conformal adaptive antenna based on multiple planar active arrays as geodesic antenna array (GEODA). This antenna is a candidate for a new generation of ground stations for satellites communications [7]. This antenna has two geometrical structure parts. The first one, is based on a cylinder conformed by 30 triangular planar active arrays (panel), and the second is a half dodecahedron geodesic dome conformed by 30 panels, as is presented in Figure 2. Each panel is consisting of 45 elements as double stacked circular patches with their own RF circuit. Finally, the panels are conformed by 15 sub-arrays (cells) with 3 elements. In total there are $45 \times(30+30)$ radiating elements.
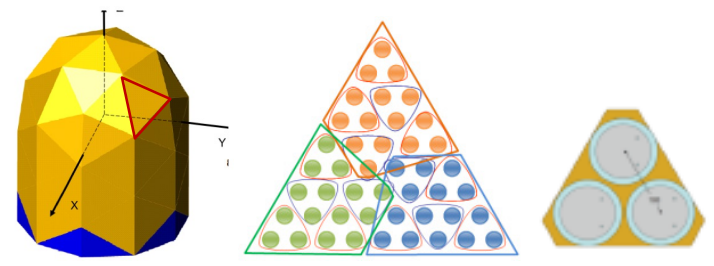

Figure 2 - GEODA structure, 45 elements planar active array (Panel) and 3 elements sub-array (Cell)

The RF circuit of each cell was made up by one hybrid coupler with an additional $25 \mathrm{~dB}$ test signal coupler for calibration purposes, an LNA with 3 states (on, off, bypass) and one phase shifter with 6 states per patch. The outputs of the 3 patches are combined into one signal using a Wilkinson combiner. This signal is amplified with another LNA at the output of the cell [7]. Consequently each panel provides 15 outputs which are combined and the resulting signal does through the RF receiver for IF conversion at $27.5 \mathrm{MHz}$.

\section{Control system}

The control system has two main parts: the hardware structure and the control system software. A three-layers architecture has been implemented for the hardware structure. The first layer consist of one embedded microcontroller (PIC18F87J10) in each cell RF circuit to control the phase shifter, the LNA and the test signal coupler switch. The second layer has a main board with one embedded microcontroller (PIC18F4550) which manages the control data address to each cell. The third layer is the work station PC which operates with the control software to manage the antenna. The control system software is able to manage one panel for its measurement, characterization and calibration. The software controls four tasks: satellite tracking, anechoic chamber measurements, $\mathrm{S}_{21}$ measurement of the RF circuit and the $S_{21}$ measurement with the test signal for RF calibration.

\section{Automated Measurements}

As discussed before, the automated measurements implemented to achiever the calibration and characterization requirements are the $S_{21}$ measurement of the RF circuits, the Test Signal measurement; and the Anechoic Chamber.

\section{1. $S_{21}$ parameter measurement of the $R F$ circuits}

For the characterization of the RF circuits, a number of measurements of the $S_{21}$ parameter must be done for the patch LNA, phase shifter, and the Cell LNA states. Figure 3 shows the setup of the automated measurement for the $S_{21}$ parameter connecting the output of the vectorial network analyzer VNA to each polarization port of the RF branch of a patch, and the input of the VNA is connected to the output of the Cell RF circuit. This test requires 4860 different measurements per triangular active array. The implemented automated procedure reduces measurement time from the 7 hours of conventional procedures to 8 minutes per cell.

\section{Test Signal measurement of the RF circuits}

The test signal is injected through the $25 \mathrm{~dB}$ coupler to characterize the RF branches including the 15 to 1 combiner an estimate the compensation matrix for the static and dynamic errors. In this test 2430 measurements must be done.

In contrast to the test 1 , the output of the vector network analyzer VNA is connected to each $25 \mathrm{~dB}$ coupler port of each cell, and the input of the VNA is connected to the output of the 15 to 1 combiner as the panel output.

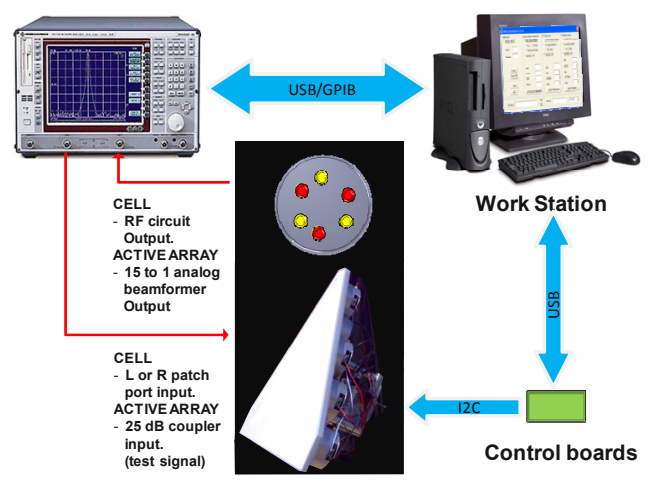

Figure 3 - S21 and the Test Signal measurement Setup

\section{Anechoic Chamber measurement}

This is probably the most expensive and important stage of the measurements. Since from calibration requirements [8], [9] and [10] this test needs almost 5 frequency and 61 measurements per frequency to fulfill the selected calibration models, the anechoic chamber measurements (305) takes with the automated system more than 9 days of nonstop work. 
Thus, according to the exhaustive required measurement campaign, an efficient and scalable automated procedure depicted in Figure 4 has been implemented to minimize operative costs and time. This procedure figured out the active pattern measurement of the $i$-th patch of each $k$-th Cell with $i$ from 1 to 3 and $k$ from 1 to 15 . Then, the radiation pattern of the Panel is measured for the desired pointing directions.

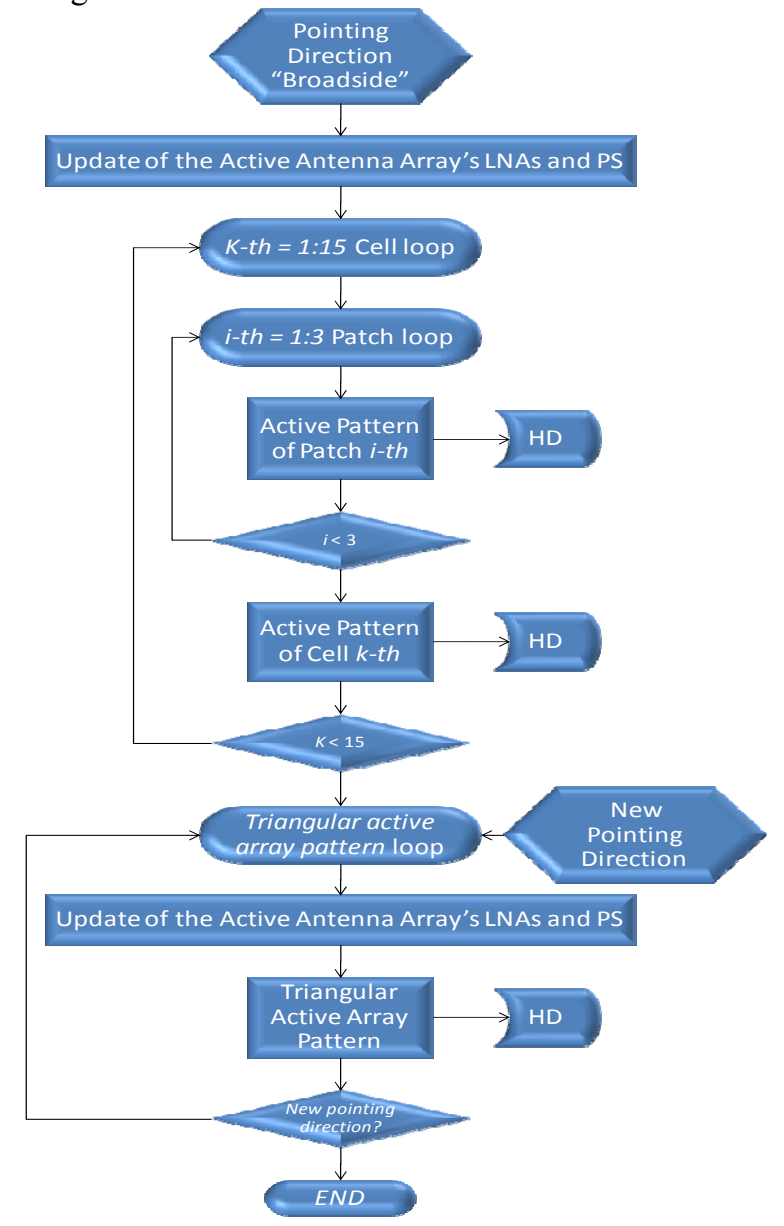

Figure 4 - Automated procedure for the anechoic chamber measurements

The measurements setup in spherical anechoic chamber is depicted in Figure 5 where the computer of the AUT (work station) is connected to the anechoic chamber computer (PC).

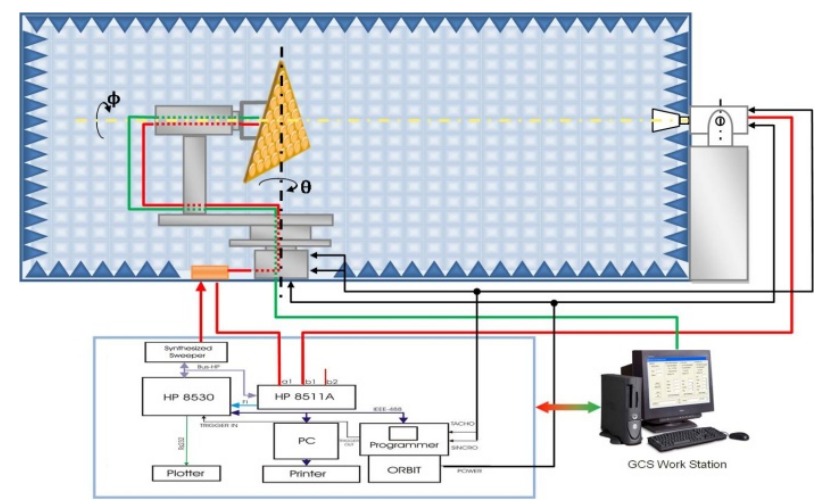

Figure 5 - Measurement setup in the spherical anechoic chamber
The work station executes the upgrade of the antenna parameters according to the inputs established by user and order to the PC to start the measurement. After each measurement the PC notify to the work station and wait for new orders. In parallel task the work station upgrade the active antenna array followed by the new measurement activation order. To measure the active pattern of one patch embedded in the active antenna array the work station has to set the rest of the patches at matched setting.

\section{RESULTS}

The laboratory and anechoic chamber measurements have been performed to complete the automated measurement procedure proposed in section 3 . The three groups of tests explained have been executed as a sequence and some of the more relevant results are presented in this discussion.

For the test of the S21 parameter of the RF circuit, it was necessary to carried out 4860 measurements of the S21 parameter to evaluate the 6 states of the phase-shifters and 3 states of the LNA of the 45 patches.

Results depicted in Figure 6 and Figure 7 have been presented to illustrate the standard deviation of the phase and gain of the RF circuits, respectively. The standard deviation of the phase presented in Fig. 7 was obtained from one cell measurements of the triangular active array, and it is a measure of the dispersion from the phase average. Furthermore, in Fig. 8 the standard deviation of the gain is showed. As previously, it is a measure of the dispersion from the gain average.

It is important to indicate that even when the results are presented as curves, the measured values are those owing to the phase shifter states as $0,60,120,180,240$ and 300 degrees.

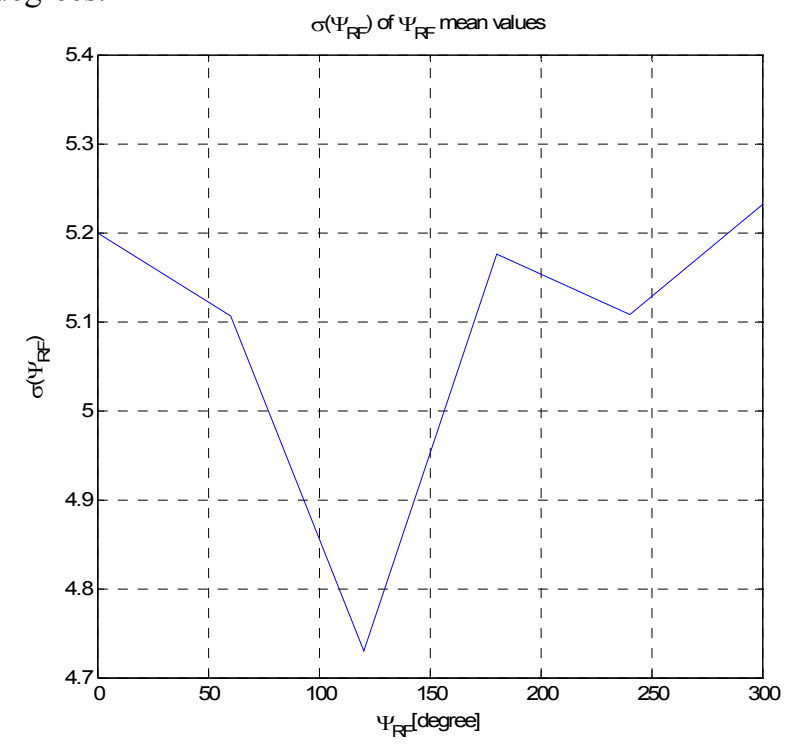

Figure 6 - Standard deviation $(\sigma)$ of the Phase of the RF circuit as a function of the phase-shifter state 


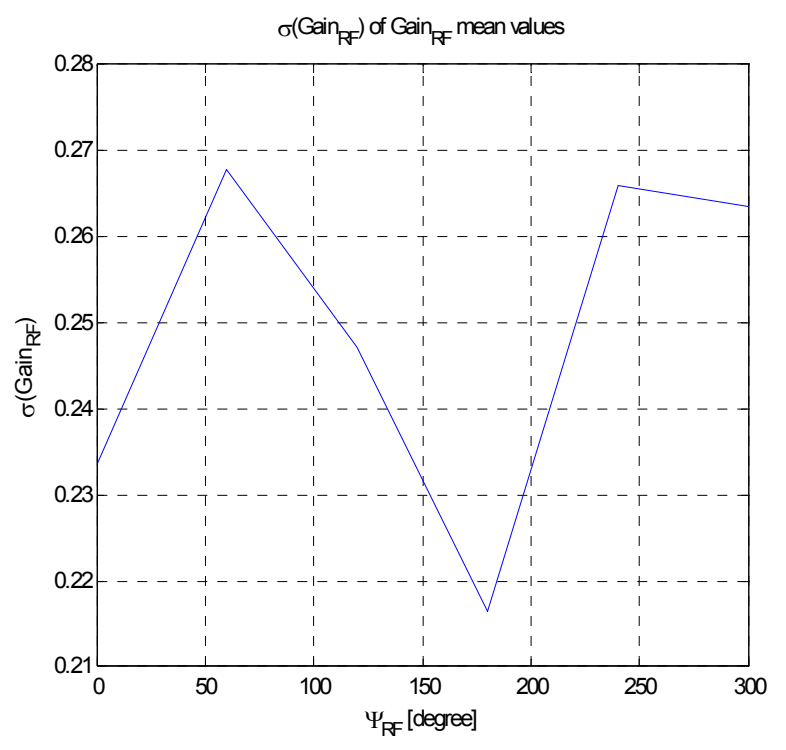

Figure 7 - Standard deviation $(\sigma)$ of the Gain of the RF circuit as a function of the phase-shifter state

With the results of the S21 measurements with the test signal, the one compensation matrix of the RF circuit which is part of the Off-line calibration procedure can be obtained. The results of the amplitude and phase of the S21 parameter of the three patches of one cell with its LNA at on are depicted in Figure 8 and Figure 9, respectively. In contrast to the first test, this representation includes the resulting weight of the RF circuit per patch from the output of the hybrid coupler to the output of the 15 to 1 combiner.

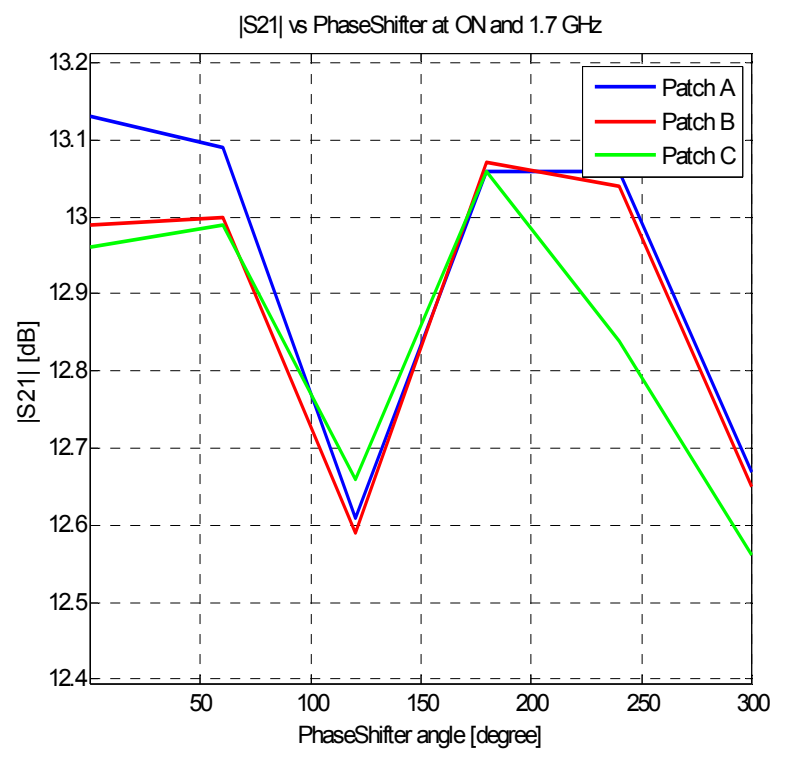

Figure 8 - $|\mathrm{S} 21|$ measured with Test signal procedure

It is easy to signify the deviation in amplitude and phase values presented in the results. The behavioral of the RF circuit components is represented in these results where the deviation performance for the phase shifters and LNAs states can be obtained and the compensation matrix computed.

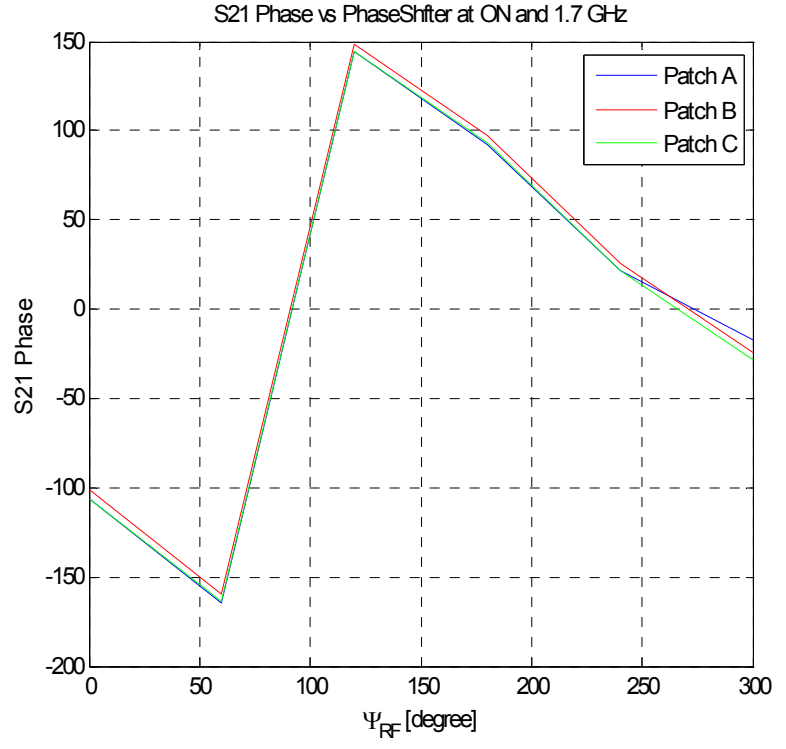

Figure 9 - S21 Phase measured with Test signal procedure

Finally, from the anechoic chamber measurement procedure we present the measured pattern of the triangular active array and the active pattern of the patches of one cell.

The Figure 10 depicts the normalized pattern of the triangular active array at theta $=30^{\circ}$, phi $=0^{\circ}$ and $\mathrm{f}=1.7$ $\mathrm{GHz}$, and the active array has a gain losses due to the misspointing about $1.5 \mathrm{~dB}$ which means a pointing error about $5^{\circ}$ for the $30^{\circ}$ pointing direction pattern presented.

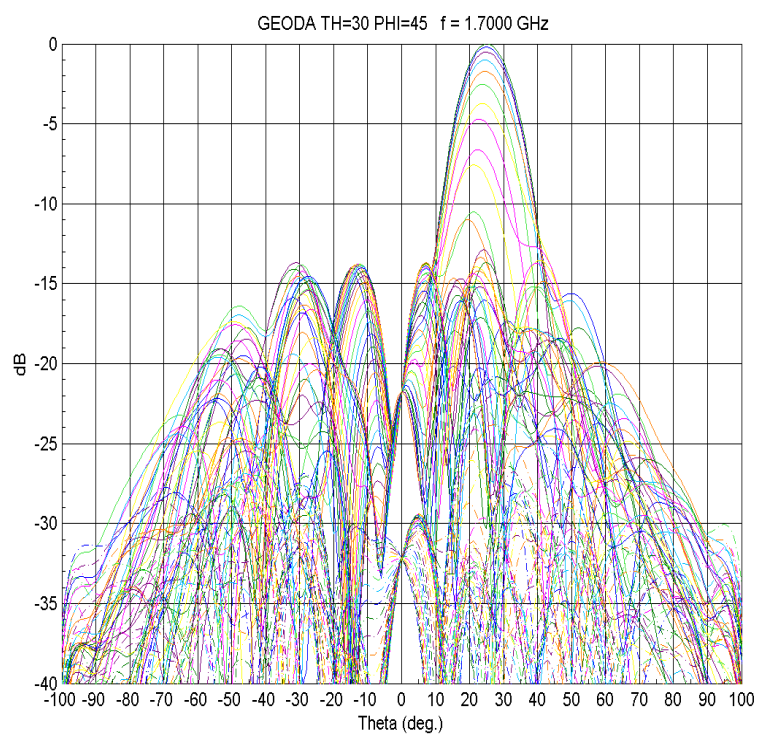

Figure 10 - Anechoic chamber measurement of the pattern of the triangular active array panel at Theta $=30^{\circ}, \mathrm{Phi}=0^{\circ}$ and $\mathrm{f}=1.7 \mathrm{GHz}$.

The active pattern for the 45 patches were measured and the Figure 11 presents the active pattern in decibels of the patches $\mathrm{A}, \mathrm{B}$ and $\mathrm{C}$ of one center Cell at $\mathrm{f}=1.7 \mathrm{GHz}$ and normalized to the broadside amplitude value. 
To obtain the compensation matrix for mutual coupling effect, it is computed as the difference between the patch pattern measured as a unique radiating element antenna and the active pattern of the patch embedded in the active array and matching the rest.

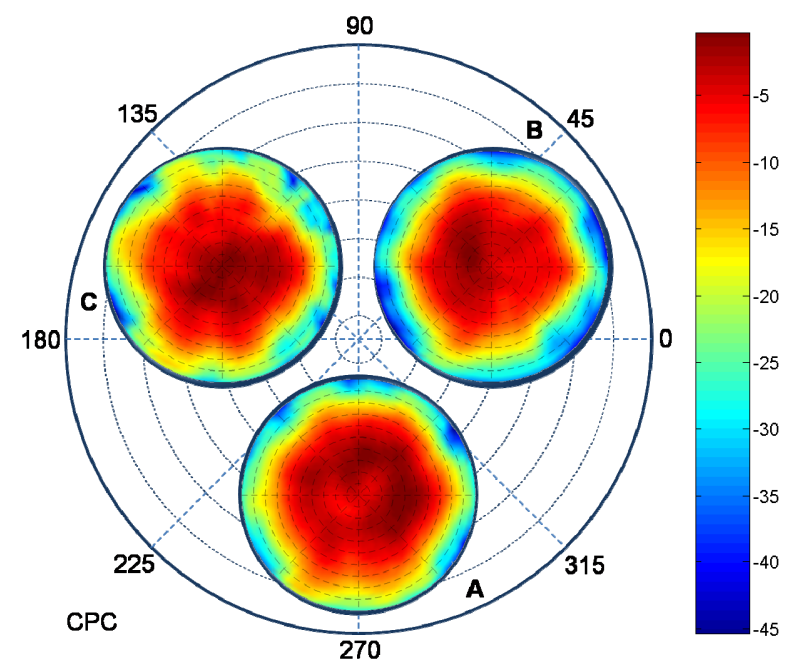

Figure 11 - Anechoic chamber measurement of the Active Pattern of the Cell 5, Patches A B C of the triangular active array panel at $\mathrm{f}=1.7 \mathrm{GHz}$.

This result shows the rippling effect of the mutual coupling in the active pattern of the three patches of one cell. This degradation must be solved applying the Off-line calibration algorithm for mutual coupling compensation.

\section{CONCLUSIONS}

In this work an automated measurement procedure for the calibration of planar active arrays is presented. Using this procedure, a reduction in measurement time is achieved thus reducing the costs required for the calibration of active antenna arrays.

First, the characterization of RF circuits has been presented. Results support that the impact of phase errors on antenna performance is larger than gain errors of the RF circuits on the gain losses for miss-pointing due to its standard deviation. Secondly, the active pattern of patch elements embedded in the array has been measured in order to model mutual coupling coefficient for off-line calibration procedures.

Finally, the required data for the calibration process from measurement has been compiled and the compensation matrices for calibration have been computed. Furthermore, anechoic chamber measurements are used to model the onsite calibration procedure to compensate the change of the work frequency for transmission.

In addition, one automatic system scalable for future active antenna array designs has been implemented and tested in the anechoic chamber.

\section{ACKNOWLEDGMENT}

Authors wish to thank MICINN (Ministerio de Ciencia e Innovación) for the goberment grant and CICYT (Comisión Interministerial de Ciencia y Tecnología) under the CROCANTE project (ref: TEC2008-06736/TEC) and INSA (Ingeniería y Servicios Aeroespaciales S.A.) for the partial funding of this work.

\section{REFERENCES}

[1] M. A. Salas Natera, R. Martínez Rodríguez-Osorio, A. Antón Sánchez, I. García-Rojo, and L. Cuellar, "A3TB: Adaptive Antenna Array test-bed for tracking LEO satellites based on software-defined radio," in Proc. 59th International Astronautical Congress, Glasgow, October 2008, pp. 313317.

[2] A. Torre, J. Gonzalo, M. Pulido, and R. Martínez RodríguezOsorio, "New generation Ground Segment Architecture for LEO satellites," in Proc. 57th International Astronautical Congress, Valencia, October 2006, pp. 221-226.

[3] W. D. Ivancic, "Architecture and System Engineering Development Study of Space-Based Networks for NASA Missions," in Proc. IEEE Aerospace Conference, Big Sky, March 2003, pp. 1179-1186.

[4] M. Arias Campo, I. Montesinos Ortego, J. L. Fernández Jambrina, and M. Sierra Pérez, "GEODA - GRUA: Diseño del módulo T/R," in Proc. XXIV Simposium Nacional de la Unión Científica de Radio, Santander, Septiembre 2009, pp. 115-116.

[5] B. Borat and B. Friedlander, "Accuracy Requirements in OffLine Array Calibration," IEEE Transactions on Aerospace and Electronic Systems, vol. 33, no. 2, pp. 545-556, Apr. 1997.

[6] J. Wout and M. Luc, "A new antenna calibration method and a selection of a measurement probe with minimal disturbance and sufficient sensitivity for electromagnetic exposure measurements around wireless base stations," in Proc. XXVIIth General Assembly of the International Union of Radio Science, Maastricht, Aug. 2002, p. 4.

[7] M. Sierra Pérez, A. Torre, J. L. Masa Campos, D. Ktorza, and I. Montesinos, "GEODA: Adaptive Antenna Array for Metop Satellite Signal Reception," in Proc. 4th ESA International Workshop on Tracking, Telemetry and Command System for Space Application, Darmstadt, September 2007, pp. 1-4.

[8] Y. Rockah and P. Schultheiss, "Array shape calibration using sources in unknown location - Part I: Far Field Sources," IEEE Transactions on Acoustic, Speech and Signal Precessing, vol. 35, no. 3, pp. 245-253, Mar. 1987.

[9] D. M. Pozar, "A relation between the active input impedance and the active element pattern of a phased array," IEEE Transactions on Antennas and Propagation, vol. 51, no. 9, pp. 2486-2489, Sep. 2003.

[10] I. J. Gupta, J. R. Baxter, S. W. Ellingson, P. Hyung-Geun, O. Hyun Seo, and K. Mun Geon, "An experimental study of antenna array calibration," IEEE Transactions on Antennas and Propagation, vol. 51, no. 3, pp. 664-667, Mar. 2003. 